\title{
Effects of the virus-like particles of a braconid endoparasitoid, Meteorus pulchricornis, on hemocytes and hematopoietic organs of its noctuid host, Pseudaletia separata
}

\author{
Masahiro Suzuki, ${ }^{\dagger}$ Ken MiUrA* and Toshiharu TANAKA \\ Laboratory of Applied Entomology, Graduate School of Bio-Agricultural Sciences, Nagoya University; Nagoya 464-8601, Japan \\ (Received 18 January 2008; Accepted 26 September 2008)
}

\begin{abstract}
Previously, we reported that virus-like particles of Meteorus pulchricornis (MpVLPs) induced apoptosis in hemocytes of its host, Pseudaletia separata, and that MpVLPs seem to especially damage the granulocyte population in vivo. The present study was conducted to elucidate more clearly the distinct behavior of two hemocyte populations in response to MpVLPs or parasitization. First, we examined the differences between plasmatocytes and granulocytes in their sensitivity to MpVLP-induced apoptosis and also in changes of their numbers after parasitization. MpVLPs induced apoptosis in both hemocyte species in vitro. The number of plasmatocytes increased slightly after parasitization in vivo unlike that of granulocytes. The BrdU incorporation assay indicated that the parasitization or the injection of venom containing MpVLPs $(\mathrm{V}(+) \mathrm{VLP})$ enhanced the proliferation of plasmatocytes and suppressed that of granulocytes, accounting in part for the distinct responses shown by the respective hemocyte populations. Next, we examined the effects of $\mathrm{V}(+) \mathrm{VLP}$ on hematopoietic organs (HPOs), major tissues for hematopoiesis of P. separata. A large number (approximately 70-80\%) of the cells released from cultured HPOs were determined to be plasmatocytes by the morphology and response to growth-blocking peptide. The injection of V(+)VLP did not affect HPOs in terms of the hematopoietic activity and hemocyte species released. These results, together with our previous data, suggest that the plasmatocyte population was maintained by both supply from undamaged HPOs and the propagation of circulating cells during the initial phase of at least $24 \mathrm{~h}$ post-parasitization, while MpVLPs caused apoptosis irrespective of hemocyte species.
\end{abstract}

Key words: Endoparasitoid wasp; virus-like particle; hemocyte; apoptosis; hematopoietic organ

\section{INTRODUCTION}

Endoparasitoid wasps regulate the physiological conditions of their hosts to ensure successful survival and development (Vinson and Iwansch, 1980). It is especially important for these parasitoids to escape host immune responses. In lepidopteran insects, foreign substances like wasp eggs or larvae are excluded by the encapsulation reaction mediated by plasmatocytes and granulocytes (Lavine and Strand, 2002). Endoparasitoid wasps protect their eggs against the host immune response by using either the venom, calyx fluid, polydnavirus (PDV), non-PDV symbiotic viruses, or virus-like particles (Pennacchio and Strand,
2006). In particular, host regulation by PDV, a symbiotic virus associated with ichneumonid and braconid endoparasitoids, has already been well-characterized. The PDV is introduced into host hemocoel along with oviposition and then expresses its genes in various infected host cells, changing the host physiological conditions to a suitable environment for endoparasitoids (Kroemer and Webb, 2004).

The endoparasitoid Meteorus pulchricornis (Wesmael) lacks PDV, unlike other braconid wasps. Instead, it produces virus-like particles (MpVLPs) in venom gland filaments, which regulate the physiological conditions of its host (Suzuki and Tanaka, 2006). M. pulchricornis has a wide host range, in-

\footnotetext{
* To whom correspondence should be addressed at: E-mail: k-miura@agr.nagoya-u.ac.jp

${ }^{\dagger}$ Present address: Department of Entomology, University of Kentucky, Lexington, KY 40506, USA.

DOI: 10.1303/aez.2009.115
} 
cluding 9 families of Lepidoptera (Marsh, 1979; Huddleston, 1980; Maetô, 1989, 1990). While this suggests that the wasp employs distinct host regulation strategies from PDV-carrying parasitoids, the host regulation mechanisms of Meteorinae wasps have yet to be determined. In a previous study, we reported that MpVLPs induced the hemocytes, especially granulocytes, to undergo apoptosis in parasitized hosts, which could be a main factor to impair the immune response by host hemocytes (Suzuki and Tanaka, 2006). The question arises here as to why a higher proportion of apoptotic cells is observed in circulating granulocytes than in plasmatocytes. In the present study, we first examined the specificity of MpVLP-induced apoptosis in plasmatocytes and granulocytes in vitro, and counted the numbers of these hemocyte species per parasitized host. The hemocyte composition in parasitized hosts is determined by the balance between MpVLP-induced cell death and hematopoiesis, which comprises the proliferation of circulating hemocytes and de novo hemocyte production by hematopoietic organs (HPOs) (Jones, 1970; Arnold and Hinks, 1976; Beaulaton, 1979; Gardiner and Strand, 2000; Yamashita and Iwabuchi, 2001; Nakahara et al., 2003; Nardi et al., 2003; Teramoto and Tanaka, 2004; Ling et al., 2005). We assessed the proliferation of the two hemocyte species after parasitization as well as the effects of MpVLPs on the HPOs. In consequence, we clarified: 1) MpVLPs induced apoptosis in both hemocyte species; 2) MpVLPs impaired granulocyte mitosis in circulation; 3) the host HPOs produced plasmatocytes as a major part irrespective of the presence or absence of MpVLPs. Taken together, these results reasonably account for the distinct responses shown by the two hemocyte species in the early stage of parasitism.

\section{MATERIALS AND METHODS}

Insects. A solitary larval endoparasitoid, Meteorus pulchricornis (Wesmael) (Braconidae: Hymenoptera) and its host, Pseudaletia separata (Walker) (Noctuidae: Lepidoptera) were reared as described in Suzuki and Tanaka (2007). Venom containing virus-like particles $(\mathrm{V}(+) \mathrm{VLP})$ and MpVLP-free venom (V(-)VLP) were prepared as described in Suzuki and Tanaka $(2006,2007)$.

Preparation of plasmatocyte-enriched or gran- ulocyte monolayers. A plasmatocyte-enriched hemocyte fraction was prepared by the method of Taniai et al. (1997) with some modifications. Day26 th instar larvae of $P$. separata $\left(\mathrm{VI}_{2}\right.$, hereafter abbreviated in the same manner) were anesthetized under $\mathrm{CO}_{2}$, sterilized with $70 \%$ ethanol, and chilled in ice-cold water. The larvae were injected with $500 \mu \mathrm{l}$ PBS containing 8\% (v/v) saturated phenylthiourea (PTU) and $4 \mathrm{~mm} 4$-(2-aminoethyl) benzenesulfonyl fluoride (AEBSF, a serine protease inhibitor) (AEBSF-PTU-PBS) via their prolegs, and then the hemolymph was collected in a $15 \mathrm{ml}$ tube. The hemolymph from four larvae was adjusted to $4 \mathrm{ml}$ with AEBSF-PTU-PBS. Hemocyte suspensions $(200 \mu \mathrm{l}$ each) were seeded into each well of an 8-well chamber slide (IWAKI). To obtain a plasmatocyte-enriched monolayer, hemocytes in each well were incubated for $15 \mathrm{~min}$ at room temperature and allowed to spread out. Each well was washed with PTU-PBS three times to discard nonadhering hemocytes and hemolymph, and then overlaid with TC-100 medium containing $1 \mu \mathrm{M}$ growth-blocking peptide (GBP). Stimulation with GBP enhanced the spreading and adhesion activities of plasmatocytes, and, in contrast, attenuated the activities of granulocytes (Strand and Clark, 1999; Strand et al., 2000). By this treatment, adhering granulocytes became detached and floated by pipetting. After further washing twice with TC-100 containing 10\% FBS (FBS-TC), the resulting plasmatocyte-enriched monolayer (approximately 80\% pure, 9,000 cells/well) was used for the following experiments. The GBP (amino acid sequence: ENFSGGCVAGYMRTPDGRCKPTF) was synthesized by Fmoc method (Sigma) according to Ohnishi et al. (1995), Strand et al. (2000) and Aizawa et al. (2001).

The granulocyte monolayer was prepared according to Tojo et al. (2000). $\mathrm{VI}_{2}$ larvae were bled on a Parafilm sheet (American Can Co.) placed on ice, and the hemolymph was immediately diluted 20 times with PTU-TES $(100 \mathrm{~mm}$ Tris-HCl, $\mathrm{pH}$ 7.0, $120 \mathrm{~mm}$ EDTA, $150 \mathrm{~mm} \mathrm{NaCl}, 8 \%$ (v/v) saturated PTU). Hemocyte suspensions (200 $\mu$ l each) were seeded into each well of an 8-well chamber slide and incubated for $30 \mathrm{~min}$ at room temperature. The granulocyte monolayer (over 95\% pure, 9,000 cells/well) was prepared by washing with PTU-PBS three times, and then with FBS-TC twice.

TUNEL assay for detecting apoptosis. Plasma- 
tocyte-enriched or granulocyte monolayers prepared on 8-well chamber slides were incubated in $200 \mu$ FBS-TC containing either 1 or $1 / 10$ female equivalent (f.e.) $\mathrm{V}(+) \mathrm{VLP}, 1$ f.e. $\mathrm{V}(-) \mathrm{VLP}$ or PBS for $16 \mathrm{~h}$ at $27^{\circ} \mathrm{C}$. Each hemocyte monolayer was fixed with $4 \%$ paraformaldehyde (PFA) in PBS. To detect DNA fragmentation as an apoptosis marker, the plasmatocytes and granulocytes were stained according to the protocol of DeadEnd ${ }^{\mathrm{TM}}$ Fluorometric TUNEL System (Promega) and observed under a fluorescence microscope (Olympus, Model BX41). We assessed 200-250 granulocytes or plasmatocytes from four to eight random fields of view. The experiments were performed in triplicate for the respective categories.

Numbers of plasmatocytes and granulocytes normalized by total hemolymph volume in parasitized hosts. The $\mathrm{V}_{0}$ larvae were parasitized, and the densities of plasmatocytes and granulocytes in 10 host larvae were counted at 24 and $48 \mathrm{~h}$ postparasitization, as described in Teramoto and Tanaka (2004).

The total hemolymph volume of the hosts was calculated according to Weinberg (1980). The hosts were injected with $5 \mathrm{mg} / \mathrm{ml}$ amaranth in PBS and bled onto a Parafilm sheet placed on ice a few minutes after injection. The hemolymph was diluted 10 times with $0.5 \%(\mathrm{w} / \mathrm{v})$ SDS in PBS, and the absorbance of the diluted hemolymph at $515 \mathrm{~nm}$ was measured immediately in a spectrophotometer (Hitachi, U-2000A). The numbers of plasmatocytes or granulocytes per host larva were calculated by multiplying the average hemocyte density by that of total hemolymph volume.

Proportion of plasmatocytes and granulocytes incorporating bromodeoxyuridine (BrdU). $\mathrm{V}_{0}$ larvae were parasitized or injected with $\mathrm{V}(+) \mathrm{VLP}$. After $12 \mathrm{~h}, \mathrm{BrdU}(500 \mathrm{ng} / \mathrm{mg}$ body weight, in PBS/DMSO (1:1)) was injected into these larvae. The circulating hemocytes were collected by injecting ice-cold anti-coagulant solution (ACS; $98 \mathrm{~mm} \mathrm{NaOH}, 186 \mathrm{~mm} \mathrm{NaCl}, 17 \mathrm{~mm}$ EDTA, $41 \mathrm{~mm}$ citric acid, $\mathrm{pH}$ 4.5) $8 \mathrm{~h}$ after BrdU injection, centrifuged at $600 \times g$ for $10 \mathrm{~min}$ at $4^{\circ} \mathrm{C}$, washed and suspended in ACS. Hemocyte suspensions were spread over a MAS-coated slide glass (Matsunami) placed on ice for $20 \mathrm{~min}$ to allow the hemocytes to settle onto the glass surface. The settled hemocytes were fixed with 4\% PFA in PBS for 10 min, permeabilized with PBS containing 0.2\% Triton X-100, exposed to $3 \mathrm{M} \mathrm{HCl}$ for $20 \mathrm{~min}$ to denature DNA, and then blocked with PBS containing $2 \%(\mathrm{w} / \mathrm{v})$ BSA and $0.01 \%(\mathrm{w} / \mathrm{v})$ Triton X-100 (BT-PBS) for $30 \mathrm{~min}$. The hemocyte preparations were overlaid with BT-PBS containing anti-BrdU antibody $(1: 50)$ for $24 \mathrm{~h}$ at $4{ }^{\circ} \mathrm{C}$. This was followed by staining with Alexa Fluor ${ }^{\circledR}$ 568-labeled secondary antibody (1:400, Invitrogen), and the preparations were observed under a fluorescence microscope after four washes in PBS. For each sample, BrdU incorporation was examined in 500 hemocytes in eight random fields of view. One sample was composed of the hemocytes collected from seven $P$. separata larvae. Three independent hemocyte preparations were examined for each category.

Morphological examination of HPO. The HPO of host larva forms a complex with a wing disc (WD) and produces hemocytes (Fig. 5A). HPOs obtained from $\mathrm{VI}_{0}$ or $\mathrm{VI}_{1}$ were used for morphological observations and quantitative analyses, since the HPOs of fifth or lower stadium instar larvae were too small for these experiments. Our preliminary experiments showed that the injection of $1 / 2$ f.e. $V(+) V L P$ into $V_{0}$ larvae or that of 1 f.e. $\mathrm{V}(+) \mathrm{VLP}$ into $\mathrm{VI}_{1}$ resulted in decreased numbers of circulating hemocytes to almost the same degree as parasitization.

Twenty-four hours after the injection of 1 f.e. $\mathrm{V}(+) \mathrm{VLP}$ into $\mathrm{VI}_{1}$ larvae, a small piece of integument with WD was clipped out, utilizing the position of the WD-HPO complex, which was visually recognized by the side colored line on the integument. Using a surgical blade, the WD-HPO was cut into TC-100 medium containing $8 \%(\mathrm{v} / \mathrm{v})$ saturated PTU. Ten HPOs were prepared, and the respective major and minor axes were measured.

Twenty-four hours after the injection of $1 / 2$ f.e. $\mathrm{V}(+) \mathrm{VLP}$ into $\mathrm{VI}_{0}$ larvae, the HPOs were collected and fixed by $4 \%$ PFA in PBS for $16 \mathrm{~h}$, dehydrated with ethanol series, embedded in a paraffin block, and cut into $8 \mu \mathrm{m}$ sections. The paraffin sections were stained with Mayer's hematoxylin and 1\% eosin $\mathrm{Y}$ solution according to the procedure described by Sano (1965).

BrdU incorporation into HPO cells. $\mathrm{VI}_{0}$ larvae were injected with BrdU $4 \mathrm{~h}$ after injection of $1 / 2$ f.e. $\mathrm{V}(+) \mathrm{VLP}$. At 10 or $20 \mathrm{~h}$ after BrdU injection, the HPOs were dissected as mentioned above, and then immersed in maceration solution (glacial acetic acid:glycerol:water, $1: 1: 15, \mathrm{v}: \mathrm{v}: \mathrm{v})$ for 
10 min according to the method of Nardi et al. (2003) to disintegrate the HPOs. The HPO cells were dispersed by vortexing, passed through $100 \mu \mathrm{m}$ mesh (BD Falcon) to remove tissue debris, and fixed on MAS-coated slide glasses with $4 \%$ PFA. The HPO cell monolayer was stained with anti-BrdU antibody. Seven hundred HPO cells in three random fields of view were assessed for BrdU incorporation. The HPO taken from six $P$. separata was used as a sample. Each category was examined in triplicate.

Amounts and types of cells discharged from HPOs during in vitro incubation. Twenty-four hours after injection of $1 / 2$ f.e. $\mathrm{V}(+) \mathrm{VLP}$ into $\mathrm{VI}_{0}$ larvae, the HPOs were dissected from the larvae, washed in TC-100 several times, and then cultured in $100 \mu \mathrm{l}$ FBS-TC containing $10 \%$ heat-treated $\left(60^{\circ} \mathrm{C}, 10 \mathrm{~min}\right)$ hemolymph in a 96 -well plate for $40 \mathrm{~h}$ at $27^{\circ} \mathrm{C}$. The amounts of cells discharged from the HPOs were measured by 3-(4,5-dimethylthiazol-2-yl)-2,5-diphenyl tetrazolium bromide (MTT) assay. Yellow MTT is reduced to purple formazan by the function of mitochondria. The amount of MTT formazan produced is proportional to the cell number (Mosmann, 1983). After removing the HPOs from each well, $20 \mu \mathrm{lof} 5 \mathrm{mg} / \mathrm{ml}(\mathrm{w} / \mathrm{v})$ MTT solutions were added to each well containing discharged cells, incubation was continued for $4 \mathrm{~h}$ at $27^{\circ} \mathrm{C}$, and then $100 \mu \mathrm{l}$ of isopropanol containing $0.04 \mathrm{M} \mathrm{HCl}$ was added to the wells. After complete dissolution of the MTT formazan, absorbance was measured at $595 \mathrm{~nm}$. The respective values of the discharged cells are shown relative to the control value set at 100. Replication was performed in 17 and 14 HPOs from V(+)VLP- and PBS-injected larvae, respectively.

Four or five HPOs collected from $\mathrm{V}(+) \mathrm{VLP}$ or PBS-injected larvae were cultured in Sf-900 II medium (Invitrogen) containing 10\% FBS in a 12well plate for $40 \mathrm{~h}$ at $27^{\circ} \mathrm{C}$. After HPO removal from each well, the discharged cells were further cultured for $24 \mathrm{~h}$ at $27^{\circ} \mathrm{C}$. The cells were then exposed to $1 \mu \mathrm{M}$ GBP for $2 \mathrm{~h}$ in order to discriminate plasmatocytes from other hemocyte species. The proportion of plasmatocytes in total cells was assessed in 4-8 random fields of view. Each category was examined in triplicate.

Statistical analysis. Student's $t$-test was used to compare two groups. To compare three or more groups, one-way ANOVA followed by Tukey's test was performed.

\section{RESULTS}

\section{Induction of apoptosis in host plasmatocytes and granulocytes by MpVLPs}

In order to measure the proportion of apoptotic plasmatocytes by MpVLPs treatment under hematopoiesis-excluded conditions, plasmatocyteenriched fractions were incubated with $\mathrm{V}(+) \mathrm{VLP}$ in vitro. Morphological observation revealed that the $\mathrm{V}(+) \mathrm{VLP}$ induced shrinkage and disintegration of the cultured plasmatocytes after $16 \mathrm{~h}$ of incubation (Fig. 1A, B). A large number of cell debrislike apoptotic bodies were also observed (Fig. 1A, B). Plasmatocytes incubated with V(+)VLP showed nucleic condensation and fragmentation, which were detected by TUNEL (Fig. 2A). The proportion of TUNEL-positive plasmatocytes after incubation with 1 f.e. or $1 / 10$ f.e. $V(+) V L P$ was approximately $30 \%$, which was significantly higher than with $\mathrm{V}(-) \mathrm{VLP}$ or PBS treatment (Fig. 2B). The granulocyte monolayer was also subjected to the same assay. The proportions of TUNEL-positive granulocytes were approximately $50 \%$ and $25 \%$ after treatment with 1 f.e. and $1 / 10$ f.e. $\mathrm{V}(+) \mathrm{VLP}$, respectively, while treatment with V(-)VLP or PBS readily resulted in approximately 15\% TUNEL-positive granulocytes (Fig. 2B). Thus, MpVLPs were shown to induce apoptosis not only in granulocytes but also in plasmatocytes under the in vitro conditions employed. We should note that the plasmatocyte-enriched monolayer used here was prepared using GBP treatment, which might influence the degree of apoptosis induction by $\mathrm{V}(+) \mathrm{VLP}$, and that the granulocyte monolayer was obtained without GBP treatment step (Figs. 1 and 2, see Materials and Methods for details). These two hemocyte preparations were thus obtained by distinct pretreatments, and we did not address the quantitative comparison of apoptosis induction between the two hemocyte species.

\section{Increased number and proliferation of plasma- tocytes in parasitized hosts}

The density of granulocytes in hemolymph fell markedly to 27.3 and $10.8 \%$ relative to the nonparasitized control at 24 and $48 \mathrm{~h}$ after parasitization, respectively. On the other hand, plasmatocyte density was maintained at a similar level to the 

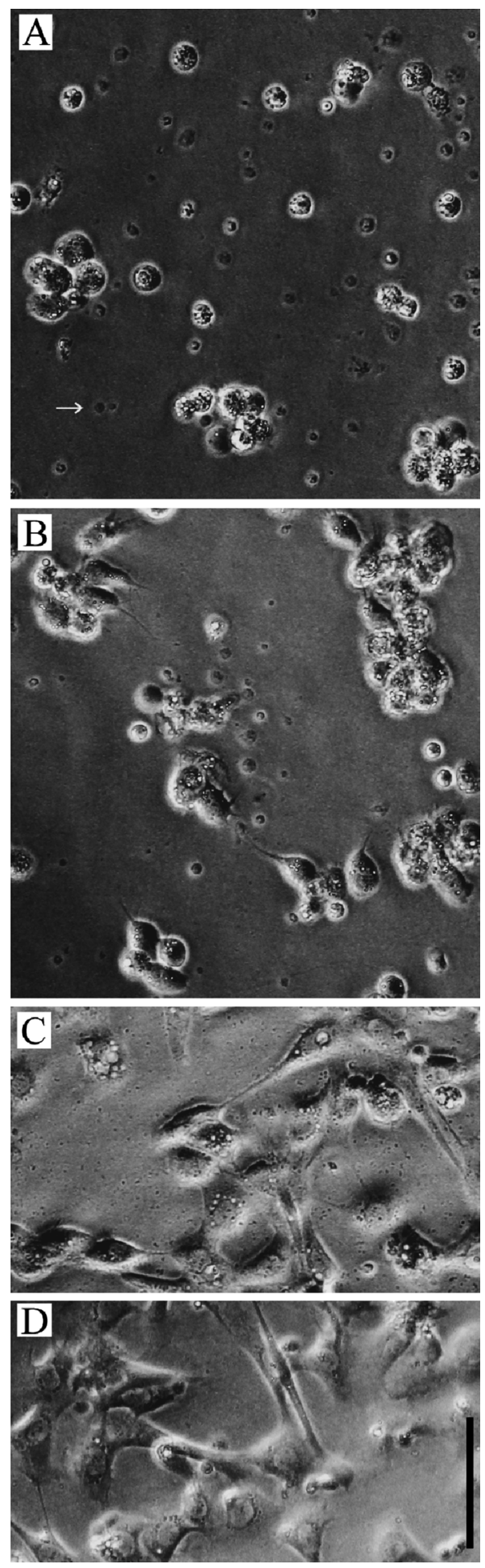

Fig. 1. Morphological changes in plasmatocytes incubated with $\mathrm{V}(+) \mathrm{VLP}$ (venom containing MpVLPs) in vitro. Plasmatocyte-enriched monolayers were incubated in $200 \mu \mathrm{l}$ TC-100 medium containing 10\% FBS with either V(+)VLP (A: 1 f.e., B: 1/10 f.e.), 1 f.e. V(-)VLP (C) or PBS (D) for 16 $\mathrm{h}$ at $27^{\circ} \mathrm{C}$. An apoptotic body is indicated by a white arrow. Scale bar represents $50 \mu \mathrm{m}$.
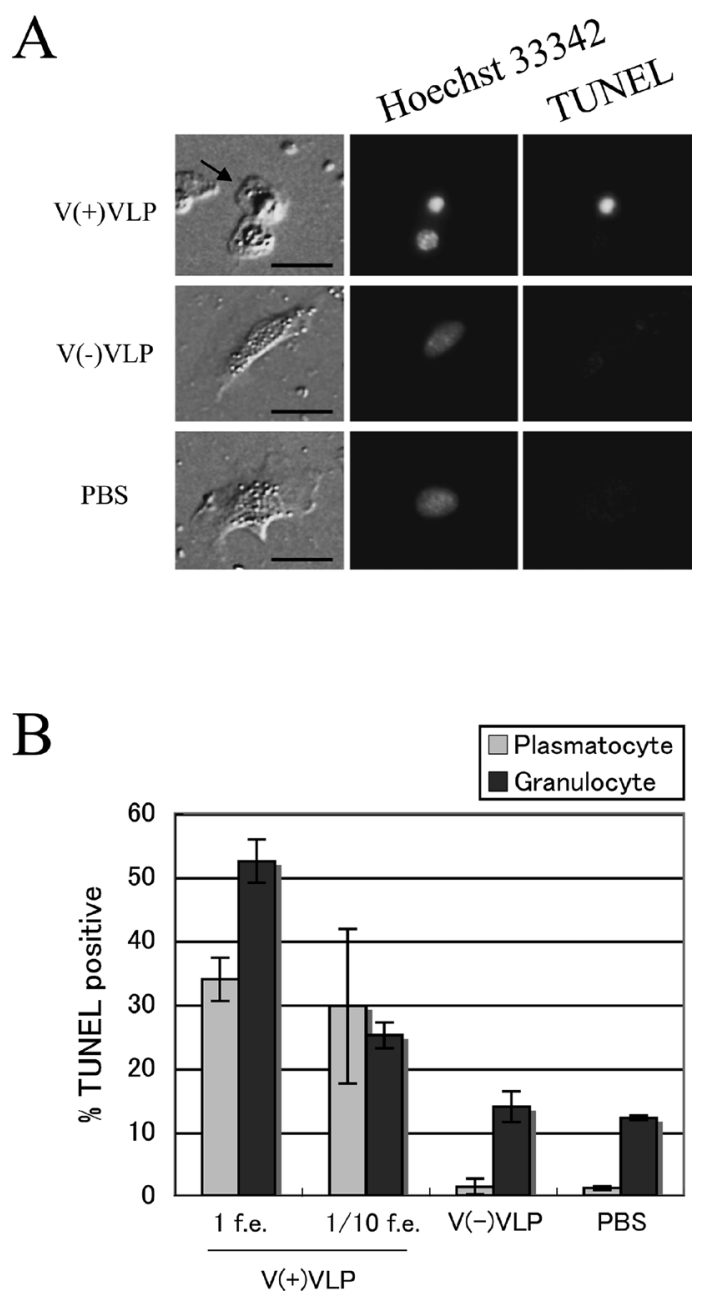

Fig. 2. TUNEL assay in hemocytes exposed to V(+)VLP in vitro. (A) TUNEL and Hoechst 33342 staining of plasmatocytes incubated under similar conditions as in Fig. 1. Nucleic condensation and fragmentation were observed in V(+)VLPtreated plasmatocytes (arrow). Scale bars represent $20 \mu \mathrm{m}$. (B) Proportion of TUNEL-positive plasmatocytes and granulocytes after incubation under the same conditions as in (A). Each category was examined in triplicate. The plasmatocyteenriched monolayer was prepared after GBP treatment while the granulocyte monolayer was obtained without GBP treatment.

control until $48 \mathrm{~h}$ after parasitization (Fig. 3). The average numbers of plasmatocytes per host larva at $24 \mathrm{~h}$ post-parasitization were 1.5 -fold higher than those at $0 \mathrm{~h}$ of the non-parasitized control, while granulocyte numbers decreased to 0.5 -fold (Table 1).

As for the proliferation of these hemocytes, while the proportion of granulocytes which incorporated BrdU in parasitized or V(+)VLP-injected host larvae was significantly lower than the control, plasmatocytes from parasitized or V(+)VLP-in- 
Table 1. Number of hemocytes per host larva

\begin{tabular}{|c|c|c|c|}
\hline $\begin{array}{c}\text { Status of larvae } \\
\text { (time after treatment) }\end{array}$ & $\begin{array}{c}\text { Hemolymph } \\
\text { volume } \pm \mathrm{SD}, \mu \mathrm{l} \\
(n)^{\mathrm{a}}\end{array}$ & $\begin{array}{l}\text { Average number of } \\
\text { plasmatocytes per } \\
\text { larva } \times 10^{5} \text { cells } \\
\text { (multiplying factor) }^{\mathrm{b}}\end{array}$ & $\begin{array}{c}\text { Average number of } \\
\text { granulocytes per larva } \\
\times 10^{5} \text { cells } \\
\text { (multiplying factor) }^{b}\end{array}$ \\
\hline \multirow{2}{*}{ Non-parasitized } & $22.7 \pm 2.4$ & 1.9 & 7.2 \\
\hline & $76.5 \pm 10.1$ & $5.2(2.7)$ & $20.5(2.8)$ \\
\hline Parasitized & $46.2 \pm 10.3$ & $3.0(1.5)$ & $3.4(0.5)$ \\
\hline
\end{tabular}

${ }^{\text {a }}$ Number of examined larvae is shown in parentheses.

${ }^{\mathrm{b}}$ Numbers in parentheses represent multiplying factors for each value when compared to $0 \mathrm{~h}$ control values.

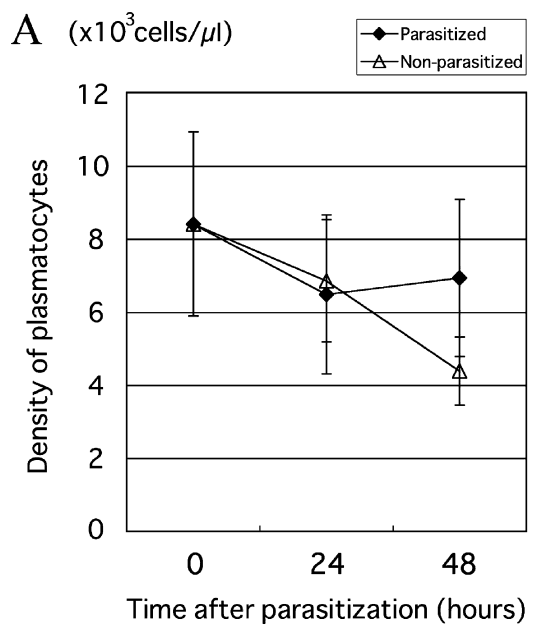

B $\left(x 10^{4}\right.$ cells $\left./ \mu l\right)$

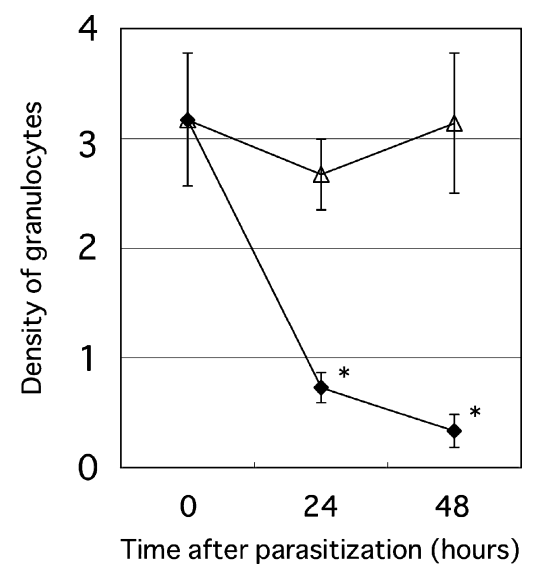

Fig. 3. Density of plasmatocyte (A) or granulocyte (B) population in parasitized hosts. Number of plasmatocytes or granulocytes per microliter of hemolymph was counted at 24 and $48 \mathrm{~h}$ post-parasitization. Asterisks represent significant differences from control values at each time point $(p<0.01)$.

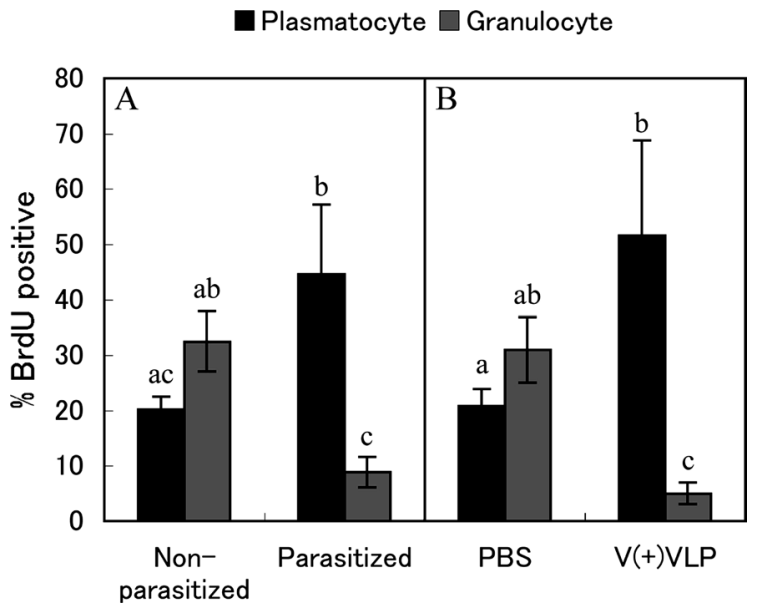

Fig. 4. Incorporation of BrdU into circulating plasmatocytes and granulocytes in parasitized (A) or V(+)VLP-injected (B) hosts. Hemocytes were collected $8 \mathrm{~h}$ after BrdU injection, which was performed $12 \mathrm{~h}$ after parasitization or $\mathrm{V}(+)$ VLP injection. Hemocytes obtained from seven larvae were used as a sample, and each category was examined in triplicate. Different letters represent significant differences at 0.05 by one-way ANOVA and Tukey's post hoc test.

jected host showed elevated levels of BrdU incorporation (Fig. 4A, B). These results indicate that the proliferation of plasmatocytes was enhanced by either parasitization or V(+)VLP injection.

\section{Effects of V(+)VLP on HPO of $P$. separata}

To estimate the contribution of the HPOs to the elevated numbers of plasmatocyte after parasitization or V(+)VLP injection, the HPOs from parasitized or $\mathrm{V}(+) \mathrm{VLP}$-injected hosts were examined further. The size of HPO, regarded as an oval, was $1.71 \pm 0.50 \mathrm{~mm}^{2} 24 \mathrm{~h}$ after $\mathrm{V}(+) \mathrm{VLP}$ injection, not differing from the control (Fig. 5A, Table 2). Tissue sections of the HPO prepared from V(+)VLPinjected host larvae showed neither morphological 

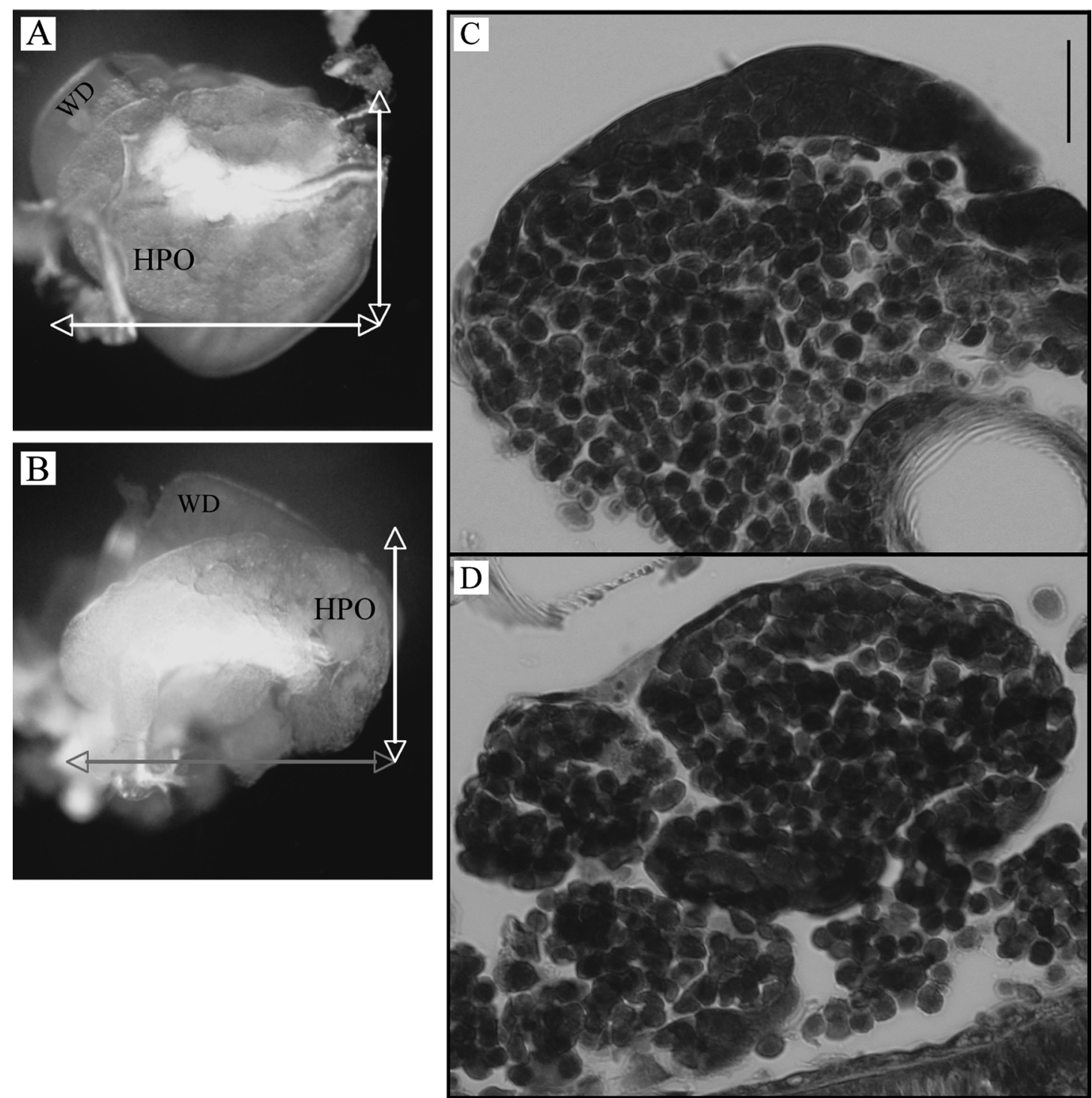

Fig. 5. Morphological observation of HPO in V(+)VLP-injected P. separata. A complex of HPO and wing disc (WD) $24 \mathrm{~h}$ after injection of PBS (A) or V(+)VLP (B). Tissue sections with hematoxylin and eosin staining are presented in C (PBS-injected control) and $\mathrm{D}(\mathrm{V}(+) \mathrm{VLP}$-injected). Scale bar represents $50 \mu \mathrm{m}$.

Table 2. Size of hematopoietic organ from MpVLP plus venom-injected P. separata

\begin{tabular}{lcccc}
\hline Injection & $\begin{array}{c}\text { Number of } \\
\text { examined } \\
\text { larvae }\end{array}$ & $\begin{array}{c}\text { Size of hematopoietic } \\
\text { organ }\left(\mathrm{mean} \pm \mathrm{SD}, \mathrm{mm}^{2}\right)\end{array}$ & $\begin{array}{c}\text { Weight of host larvae } \\
(\mathrm{mean} \pm \mathrm{SD}, \mathrm{mg})\end{array}$ & $\begin{array}{c}\text { Size } / \mathrm{weight} \\
\left(\mathrm{mean} \pm \mathrm{SD}, \mathrm{mm}^{2} / \mathrm{g}\right)\end{array}$ \\
\hline V(+)VLP & 10 & $1.71 \pm 0.50^{\mathrm{a}}$ & $557 \pm 55^{\mathrm{a}}$ & $3.08 \pm 0.86^{\mathrm{a}}$ \\
PBS & 10 & $2.00 \pm 0.36^{\mathrm{a}}$ & $598 \pm 66^{\mathrm{a}}$ & $2.55 \pm 0.38^{\mathrm{a}}$ \\
\hline
\end{tabular}

\footnotetext{
${ }^{a}$ No significant difference in each column by $t$-test.
} 
abnormality (Fig. 5B) nor TUNEL-positive cells (data not shown).

The proportion of BrdU incorporation into HPO cells was similar for PBS- or V(+)VLP-injected groups at the respective time points (Fig. 6), indicating that mitotic activity in HPO cells was affected by neither MpVLPs nor venom in an early stage of parasitism. Injection of $\mathrm{V}(+) \mathrm{VLP}$ also had no significant effect on the ability of HPOs to dis-

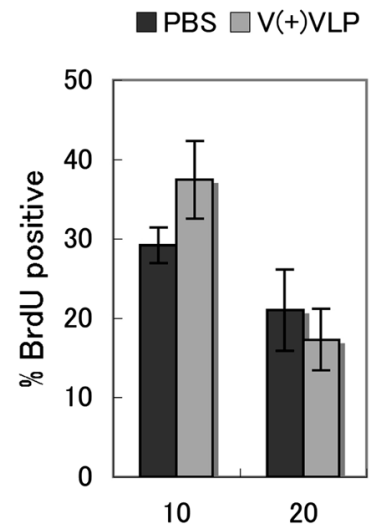

Time after BrdU injection (hours)

Fig. 6. Incorporation of BrdU into HPO cells after $\mathrm{V}(+) \mathrm{VLP}$ injection. P. separata larvae were injected with BrdU $4 \mathrm{~h}$ after either $\mathrm{V}(+) \mathrm{VLP}$ or PBS injection. HPOs were dissected and examined 10 or $20 \mathrm{~h}$ after BrdU injection as described in Materials and Methods. HPO cells collected from 6 larvae were used as a sample and each category was examined in triplicate.

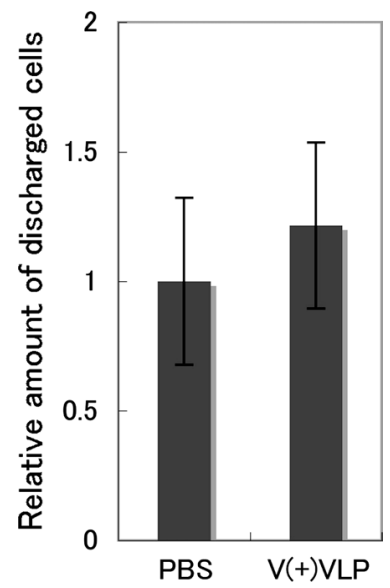

Fig. 7. Relative amount of cells discharged from HPO in vitro culture. HPOs were obtained from $P$. separata $24 \mathrm{~h}$ after $\mathrm{V}(+)$ VLP injection. Cell numbers were counted after $40 \mathrm{~h}$ incubation and presented relative to the PBS control. No significant difference was detected between PBS- $(n=14)$ and $\mathrm{V}(+)$ VLP-injected groups $(n=17)$. charge new hemocytes (Fig. 7). A large number of cells discharged from HPO exhibited plasmatocyte-like morphology and spreading behavior by GBP stimulation, indicating that these cells were
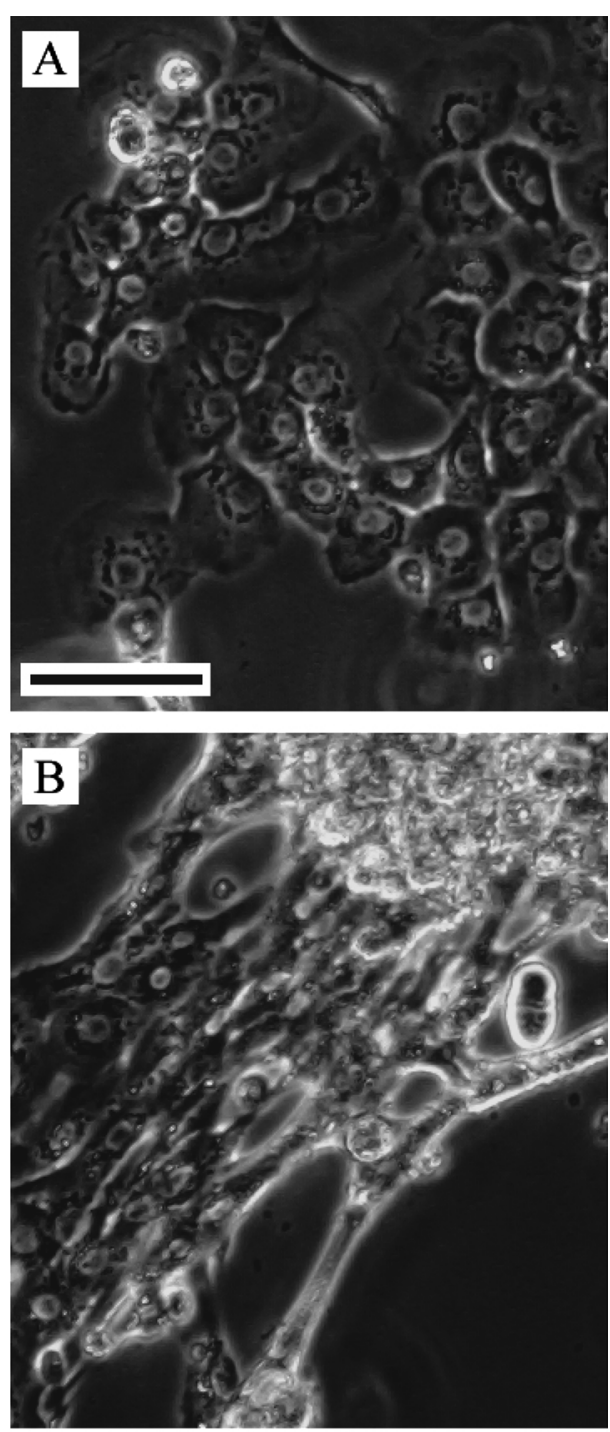

C

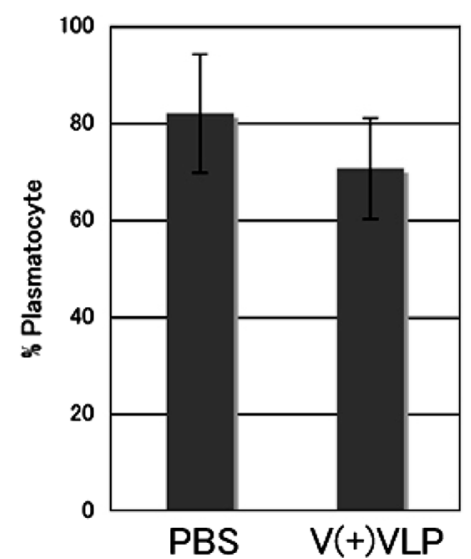


newly generated plasmatocytes (Fig. 8A, B). Moreover, V(+)VLP injection had no significant effect on the proportion of plasmatocytes (approximately 70-80\%) among the discharged cells (Fig. 8C). The remainder seemed to be undifferentiated prohemocytes, judging from their morphology.

\section{DISCUSSION}

Virus-like particles of $M$. pulchricornis induce host hemocyte apoptosis and decrease the granulocyte population for the initial two days (Suzuki and Tanaka, 2006). When M. pulchricornis parasitizes $\mathrm{IV}_{0}$ P. separata, its eggs hatch two days after parasitization (Suzuki and Tanaka, 2007). These results suggest that MpVLPs are an important factor to protect eggs from the host encapsulation response. In this study, we showed that MpVLPs induced apoptosis in plasmatocytes as well as in granulocytes. It has already been reported that PDVs induce apoptosis in host hemocytes (Strand and Pech, 1995; Teramoto and Tanaka, 2004; Luo and Pang, 2006). According to Strand and Pech (1995), granulocytes specifically undergo apoptosis by PDVs, suggesting that MpVLPs and PDVs might employ distinct mechanisms to induce apoptosis in host hemocytes.

While the proportion of apoptotic plasmatocytes was significantly lower than that of granulocytes in parasitized hosts (Suzuki and Tanaka, 2006), the present study showed that MpVLPs induced apoptosis in both plasmatocytes and granulocytes in vitro. The results obtained in this study also showed that the number of plasmatocytes per host had even slightly increased by $24 \mathrm{~h}$ post-parasitization. In parallel, the plasmatocytes exhibited higher BrdU incorporation activity. Based on these re-

Fig. 8. Hemocyte species discharged from $P$. separata HPOs. (A) Hemocytes released from normal P. separata HPOs. HPOs were cultured for $24 \mathrm{~h}$. The released cells differentiated during further incubation for $40 \mathrm{~h}$. (B) Hemocytes in the same view as in (A) were treated with $1 \mu \mathrm{M}$ GBP for $2 \mathrm{~h}$ in order to induce plasmatocyte spreading (Strand and Clark, 1999; Strand et al., 2000). Scale bar represents $50 \mu \mathrm{m}$. (C) Proportion of plasmatocytes in cells released from HPOs prepared from PBS- or V(+)VLP-injected $P$. separata. One sample contained the hemocytes released from 4-5 HPOs, and three independent samples were assessed in each category. Cells released from HPOs of PBS- or V(+)VLP-injected larvae showed no morphological differences from those of normal larvae. sults, it seems that the hematopoietic activity of plasmatocytes is temporally enhanced by parasitization or $\mathrm{V}(+) \mathrm{VLP}$ injection. In lepidopteran insects, the numbers and composition of circulating hemocyte are maintained by both the release of new hemocytes from HPOs and the mitosis of circulating hemocytes (Jones, 1970; Arnold and Hinks, 1976; Beaulaton, 1979; Gardiner and Strand, 2000; Yamashita and Iwabuchi, 2001; Nakahara et al., 2003; Nardi et al., 2003; Teramoto and Tanaka, 2004; Ling et al., 2005). The HPO is a major tissue for hemocyte production as well in $P$. separata (Teramoto and Tanaka, 2004). The present study showed that a large number of cells discharged from HPOs of $P$. separata comprised plasmatocytes, which is also the case for studies of other lepidopteran insects (Gardiner and Strand, 2000; Nakahara et al., 2003; Nardi et al., 2003). We showed in this study that V(+)VLP had no effect on HPOs in terms of morphology and hematopoietic characteristics, suggesting that undamaged HPOs contributed to the increment in plasmatocyte population after $\mathrm{V}(+) \mathrm{VLP}$ injection. Moreover, in V(+)VLP-injected larvae, plasmatocytes showed higher BrdU incorporation activity, in spite of their unchanged HPO activity, than in PBS-injected larvae, suggesting that V(+)VLP enhanced the frequency of circulating plasmatocytes to divide and to differentiate from other cell types. It is reasonable to consider that the increase of plasmatocyte population by $\mathrm{V}(+) \mathrm{VLP}$ resulted from both the release of plasmatocytes produced by undamaged HPOs and the propagation of circulating hemocytes. In contrast to the $\mathrm{V}(+) \mathrm{VLP}$, Cotesia kariyai PDV impairs HPOs (Teramoto and Tanaka, 2004), suggesting that MpVLPs and PDVs represent distinct effectors of host regulation.

The tissues where granulocytes of $P$. separata proliferate and differentiate remain to be determined. Circulating hemocytes propagate by mitosis independently of HPOs in Pseudoplusia includens and Bombyx mori during late larval stages (Gardiner and Strand, 2000; Ling et al., 2003). Thus, the granulocyte population would be maintained by mitosis of granulocytes themselves or differentiation from prohemocytes in P. separata hemocoel, which seemed to be seriously damaged by MpVLPs.

In Lepidoptera, both plasmatocytes and granulocytes are essential for successful encapsulation. In particular, granulocytes are implicated in the first 
recognition of foreign substances, and plasmatocytes can not exclude foreign substances, such as parasitoids, by themselves (Lavine and Strand, 2002). The present study together with our previous data demonstrated clearly that the parasitism of M. pulchricornis mainly resulted in impaired granulocyte population, thereby enabling the parasitoid to escape encapsulation.

The differential outcomes depending on hemocyte species, shown in this study, are unique among the wasp-host configurations reported to date (Stettler et al., 1998). At present, we can not explain why plasmatocytes increase in parasitized or $\mathrm{V}(+) \mathrm{VLP}$-injected hosts by M. pulchricornis. Since total hemocyte numbers in parasitized hosts decrease at an early phase of parasitism and do not recover throughout parasitism (Suzuki and Tanaka, 2007), the elevation of the plasmatocyte population observed in this study is likely to be a transient phenomenon specific to the early stage of parasitism. In $P$. includens, plasmatocytes show more contribution to the production of antimicrobial peptides in vivo than granulocytes (Lavine et al., 2005). The persisting plasmatocyte population in the parasitized host might function as an effector against other invaders such as bacteria and fungi.

\section{ACKNOWLEDGEMENTS}

We thank M. A. M. Bautista for reading the manuscript. This work was supported in part by a Grant-in-Aid for Scientific Research Work from the Ministry of Education, Culture, Sports, Science and Technology of Japan.

\section{REFERENCES}

Aizawa, T., Y. Hayakawa, A. Ohnishi, N. Fujitani, K. D. Clark, M. R. Strand, K. Miura, N. Koganesawa, Y. Kumaki, M. Demura, K. Nitta and K. Kawano (2001) Structure and activity of the insect cytokine growth-blocking peptide. Essential regions for mitogenic and hemocyte-stimulating activities are separate. J. Biol. Chem. 276: 3181331818.

Arnold, J. W. and C. F. Hinks (1976) Haemopoiesis in Lepidoptera. I. A note on the multiplication of spherule cells and granular haemocytes. Can. J. Zool. 61: 275-277.

Beaulaton, J. (1979) Hemocytes and hemocytopoiesis in silkworms. Biochimie 61: 157-164.

Gardiner, E. M. and M. R. Strand (2000) Hematopoiesis in larval Pseudoplusia includens and Spodoptera frugiperda. Arch. Insect Biochem. Physiol. 43: 147-167.

Huddleston, T. (1980) A revision of the western Palaearctic species of the genus Meteorus (Hymenoptera: Braconidae). Bull. Br. Mus. 41: 1-58.

Jones, J. C. (1970) Hemocytopoiesis in insects. In Regula- tion of Hematopoiesis (A. S. Gordon, ed.). Appleton Press, New York, pp. 7-65.

Kroemer, J. A. and B. A. Webb (2004) Polydnavirus genes and genomes: emerging gene families and new insights into polydnavirus replication. Annu. Rev. Entomol. 49: 431-456.

Lavine, M. D. and M. R. Strand (2002) Insect hemocytes and their role in immunity. Insect Biochem. Mol. Biol. 32: 1295-1309.

Lavine, M. D., G. Chen and M. R. Strand (2005) Immune challenge differentially affects transcript abundance of three antimicrobial peptides in hemocytes from the moth Pseudoplusia includens. Insect Biochem. Mol. Biol. 35: 1335-1346.

Ling, E., K. Shirai, R. Kanekatsu, Y. Kobayashi, T. Zhen-Li, T. Funayama, H. Watanabe and K. Kiguchi (2003) Why does hemocyte density rise during the wandering stage of the silkworm, Bombyx mori? J. Insect Biotechnol. Sericol. 72: 101-109.

Ling, E., K. Shirai, R. Kanekatsu and K. Cauchy (2005) Hemocyte differentiation in the hematopoietic organs of the silkworm, Bombyx mori: prohemocytes have the function of phagocytosis. Cell Tissue Res. 320: 535-543.

Luo, K. and Y. Pang (2006) Spodoptera litura multicapsid nucleopolyhedrovirus inhibits Microplitis bicoloratus polydnavirus-induced host granulocytes apoptosis. $J$. Insect. Physiol. 52: 795-806.

Maetô, K. (1989) Systematic studies on the tribe Meteorini (Hymenoptera, Braconidae) from Japan V. The pulchricornis group of genus Meteorus (1). Jpn. J. Entomol. 57: 581-595.

Maetô, K. (1990) Phylogenetic relationships and host associations of the subfamily Meteorinae Cresson (Hymenoptera, Braconidae). Jpn. J. Entomol. 58: 383-396.

Marsh, P. M. (1979) The braconid (Hymenoptera) parasites of the gypsy moth, Lymantria dispar (L.) (Lepidoptera: Lymantriidae). Ann. Entomol. Soc. Am. 72: 794-810.

Mosmann, T. (1983) Rapid colorimetric assay for cellular growth and survival: application to proliferation and cytotoxicity assays. J. Immunol. Meth. 65: 55-63.

Nakahara, Y., Y. Kanamori, M. Kiuchi and M. Kamimura (2003) In vitro studies of hematopoiesis in the silkworm: cell proliferation in and hemocyte discharge from the hematopoietic organ. J. Insect. Physiol. 49: 907916.

Nardi, J. B., B. Pilas, E. Ujhelyi, K. Garsha and M. R. Kanost (2003) Hematopoietic organs of Manduca sexta and hemocyte lineages. Dev. Genes Evol. 213: 477-491.

Ohnishi, A., Y. Hayakawa, Y. Matsuda, K. W. Kwon, T. A. Takahashi and S. Sekiguchi (1995) Growth-blocking peptide titer during larval development of parasitized and cold-stressed armyworm. Insect Biochem. Mol. Biol. 25: 1121-1127.

Pennacchio, F. and M. R. Strand (2006) Evolution of developmental strategies in parasitic hymenoptera. Annu. Rev. Entomol. 51: 233-258.

Sano, Y. (1965) Histological Techniques. Nanzando Company, Tokyo, pp. 179-180.

Stettler, P., T. Trenczek, T. Wyler, R. Pfister-Wilhelm and B. 
Lanzrein (1998) Overview of parasitism associated effects on host haemocytes in larval parasitoids and comparison with effects of the egg-larval parasitoid Chelonus inanitus on its host Spodoptera littoralis. J. Insect. Physiol. 44: 817-831.

Strand, M. R. and K. D. Clark (1999) Plasmatocyte spreading peptide induces spreading of plasmatocytes but represses spreading of granulocytes. Arch. Insect Biochem. Physiol. 42: 213-223.

Strand, M. R. and L. L. Pech (1995) Microplitis demolitor polydnavirus induces apoptosis of a specific haemocyte morphotype in Pseudoplusia includens. J. Gen. Virol. 76: 283-291.

Strand, M. R., Y. Hayakawa and K. D. Clark (2000) Plasmatocyte spreading peptide (PSP1) and growth blocking peptide (GBP) are multifunctional homologs. J. Insect Physiol. 46: 817-824.

Suzuki, M. and T. Tanaka (2006) Virus-like particles in venom of Meteorus pulchricornis induce host hemocyte apoptosis. J. Insect. Physiol. 52: 602-611.

Suzuki, M. and T. Tanaka (2007) Development of Meteorus pulchricornis and regulation of its noctuid host,
Pseudaletia separata. J. Insect. Physiol. 53: 1072-1078. Taniai, K., H. Wago and M. Yamakawa (1997) In vitro phagocytosis of Escherichia coli and release of lipopolysaccharide by adhering hemocytes of the silkworm, Bombyx mori. Biochem. Biophys. Res. Commun. 231: 623-627.

Teramoto, T. and T. Tanaka (2004) Mechanism of reduction in the number of the circulating hemocytes in the Pseudaletia separata host parasitized by Cotesia kariyai. J. Insect. Physiol. 50: 1103-1111.

Tojo, S., F. Naganuma, K. Arakawa and S. Yokoo (2000) Involvement of both granular cells and plasmatocytes in phagocytic reactions in the greater wax moth, Galleria mellonella. J. Insect Physiol. 46: 1129-1135.

Vinson, S. B. and G. F. Iwansch (1980) Host regulation by insect parasitoids. Q. Rev. Biol. 55: 143-165.

Weinberg, H. L. (1980) Hemolymph volume determination in the tomato fruitworm, Heliothis zea. Experientia 36: 548-549.

Yamashita, M. and K. Iwabuchi (2001) Bombyx mori prohemocyte division and differentiation in individual microcultures. J. Insect Physiol. 47: 325-331. 